FORMATION Formation emploi

Revue française de sciences sociales

97 | janvier-mars 2007

Former pour dynamiser les territoires

\title{
Petites entreprises et territoire, un lien surestimé ?
}

Small firms and the territory: an overestimated link?

Kleinunternehmen und Region?

\section{Stéphane Michun}

\section{CpenEdition}

\section{Journals}

Édition électronique

URL : http://journals.openedition.org/formationemploi/1678

DOI : $10.4000 /$ formationemploi.1678

ISSN : 2107-0946

Éditeur

La Documentation française

Édition imprimée

Date de publication : 1 janvier 2007

Pagination : $37-49$

ISSN : 0759-6340

Référence électronique

Stéphane Michun, " Petites entreprises et territoire, un lien surestimé ?», Formation emploi [En ligne], 97 | janvier-mars 2007, mis en ligne le 31 mars 2009, consulté le 30 octobre 2020. URL : http://

journals.openedition.org/formationemploi/1678; DOI : https://doi.org/10.4000/formationemploi. 1678

(C) Tous droits réservés 


\section{Petites entreprises et territoire, un lien surestimé ?}

Stéphane Michun*

Face à l'incapacité du système de formation continue à réduire les inégalités d'accès à la formation, l'idée s'est imposée qu'il suffirait de décentraliser au plus près du terrain. Pourtant, la simple proximité géographique n'est pas en mesure, à elle seule, de faire face aux spécificités et à la diversité des petites entreprises.

Une certaine idée de la compétitivité semble s'être imposée à l'ensemble des firmes, petites ou grandes, publiques ou privées. La concurrence par les prix ne disparaît évidemment pas mais elle doit composer avec les impératifs d'un régime d'innovation permanente valorisant la qualité, la variété et la réactivité. L'ensemble des entreprises doit tenir compte et, si possible, maitriser les ressorts les plus modernes de la compétitivité. Mais ce consensus sur la nature de la compétition économique ne débouche pas sur une uniformisation des entreprises, contrairement à ce que l'on avait anticipé durant la période de forte croissance. Non seulement la diversité s'accroît dans le système productif, mais les petites entreprises (nous entendons par là, en première analyse, les entreprises juridiquement indépendantes de moins de cinquante salariés) qui semblaient condamnées à disparaître ou, à la rigueur, à végéter dans quelques recoins du système productif, ont connu un regain d'intérêt indiscutable, notamment chez les décideurs politiques désireux de développer l'emploi aux différents échelons territoriaux. Il n'est pourtant pas question de passer d'un extrême à l'autre, en faisant de la petite entreprise la panacée à tous les problèmes actuels. On ne saurait nier l'importance et l'influence majeure des grandes entreprises qui semblaient appelées à une hégémonie absolue et qui continuent, aujourd'hui encore, à renforcer leur emprise dans

* Stéphane Michun est docteur en sciences économiques et ingénieur d'études au centre associé au Céreq de Montpellier, UMR 5045 «Mutations des Territoires en Europe ». Ses derniers travaux portent sur la conceptualisation des petites entreprises, à travers notamment leurs pratiques de recrutement et de formation. II a notamment publié : Michun S. (2006) (Dir.), Les métiers du tourisme. Approche régionale, Rapport pour le ministère délégué au Tourisme, Céreq, Net.Doc, nº 23 ; Bentabet M., Michun $\mathrm{S}$. et alii (2005), "Les organismes paritaires collecteurs agréés, acteurs du changement des comportements de formation des petites entreprises, Céreq ", Relief, $n^{0} 11$, décembre ; Michun S. (2003), "Les petites entreprises, des tremplins pour les jeunes débutants? », in Entreprises et jeunes débutants, sous la direction de LochetJ.F, L'Harmattan, Collection "Dynamiques d'entreprises", pp. 129-153. 
maints secteurs. De même, on ne saurait faire l'impasse sur les statistiques indiquant que la plupart des petites entreprises ont pris du retard en matière de formation, de recherche-développement et, plus largement, d'investissement immatériel. Enfin, sur le marché du travail, les petites entreprises, considérées une par une, sont en situation souvent délicate.

La petite entreprise est d'ailleurs communément présentée comme une firme par certains côtés incomplète, reposant plus sur des individus que sur une structure fonctionnelle digne de ce nom. Et, en effet, ces firmes ne sont que très rarement de grandes entreprises en miniature : l'action y est certes organisée mais cela ne se matérialise pas obligatoirement par une organisation formalisée. D'ailleurs, l'incomplétude relative de ces firmes a alimenté toutes sortes de discours et de politiques associant, comme une évidence, petites entreprises et développement local. En s'appuyant sur ces entreprises et en favorisant leur maillage, on pourrait impulser un développement endogène apportant aux territoires emplois, stabilité et solidarité (thématique du développement durable). Inversement, en s'appuyant sur le territoire, on pourrait aider efficacement les petites entreprises à se moderniser et à croître (thématique des " guichets » ou des services de proximité à destination des dirigeants). Certes, d'innombrables entreprises sont amenées à prendre appui sur les potentialités de leur environnement local afin d'améliorer la résolution de nombreux problèmes d'information, d'innovation, d'organisation du travail... Mais ne conclut-on pas un peu hâtivement à la primauté du territoire ?

À ce titre, l'exemple de la formation professionnelle continue (FPC) est assez révélateur. Face à l'incapacité du système de FPC français (construit autour d'une obligation de dépense et d'une ambition de mutualisation financière) à réduire significativement les inégalités d'accès à la formation au profit, notamment, des salariés des petites entreprises, l'idée s'est imposée qu'il suffirait de se rapprocher de ces entreprises en décentralisant au plus près du terrain les structures chargées de les accompagner dans la « modernisation» de leurs pratiques d'acquisition, de développement et de renouvellement des ressources humaines. Nous démontrerons que la simple proximité géographique n'est pas en mesure, à elle seule, de faire face aux spécificités et à la diversité des petites entreprises. La forêt des petites entreprises « traditionnelles » ne doit pas cacher les toujours plus nombreuses petites entreprises «managériales » et, subsidiairement, les plus rares petites entreprises «néolibérales». Or, ces trois manifestations d'un même format d'entreprise obéissent à des mobiles et à des règles de fonctionnement différents, ce qui induit une pluralité de positionnements par rapport au territoire et aux réseaux. Les petites entreprises traditionnelles elles-mêmes, bien que fondées sur un modèle de proximité, ne se laissent pas approcher aussi facilement par les intermédiaires de l'emploi et de la formation et ne trouvent pas toujours satisfaction dans les « guichets » et les divers « services de proximité » censés les concerner. C'est d'ailleurs sur ces dernières entreprises, de loin les plus fréquentes, que nous nous arrêterons afin de plaider la cause des réseaux «affinitaires », leviers essentiels de toute politique ambitieuse en faveur des laissés pour compte du système de formation professionnelle continue.

\section{L'ANCRAGE LOCAL, UNE SOLUTION PARMI D'AUTRES}

Une des caractéristiques majeures d'une organisation, c'est qu'elle est plus que la somme des parties qui la composent et que, partant, elle ne change ni de nature ni de forme lorsque l'on y ajoute ou retranche un élément (un salarié, un client, un fournisseur, un investissement, etc.) Dès lors, la plupart des petites entreprises ne sont pas des organisations achevées et ne le seront peut-être jamais. Ce sont des quasiorganisations devant réduire les incertitudes qui pèsent sur elles et contourner leur incomplétude relative en prenant appui sur leur environnement (Michun, 2000). Divers ancrages sont alors envisageables: une immersion dans le territoire local bien sûr, mais aussi un accrochage à des groupes ou réseaux d'entreprises développant leur activité dans des espaces plus vastes ou bien encore une inscription dans un marché professionnel du travail.

\section{Une quasi-organisation qui se « coule » dans son environnement}

En tant que quasi-organisation, la petite entreprise se construit et évolue d'abord en fonction du profil, des 
préférences et des objectifs de son dirigeant. Pour autant, chacun des collaborateurs et salariés est important et contribue, dans une certaine mesure, à l'identité de la petite entreprise car les écrans entre l'individu et l'entreprise (fonctions, services, départements, syndicats, etc.) n'existent pas ou sont peu développés. De même, les écrans entre la petite entreprise et son environnement sont souvent ténus : les relations individualisées, sans intermédiaire, sont privilégiées. La petite entreprise est, en tant que quasi-organisation, particulièrement vulnérable et/ou dépendante de ses fournisseurs, «partenaires » (donneurs d'ordres, sous-traitants, organismes de recherche, appareil de formation, etc.), concurrents, apporteurs de fonds, et clients... Mais cet environnement peut aussi lui offrir toutes sortes d'opportunités, d'appuis, de ressources et d'informations. Il faut donc reconnaître, à côté de l'influence individuelle du dirigeant, le rôle déterminant joué par l'interactivité aussi bien en interne (vis-à-vis des membres de l'équipe) qu'à l'égard de «l'extérieur». La notion de « contrôlabilité » appliquée par Guilhon aux PME (petites et moyennes entreprises) nous semble, par conséquent, devoir être reprise. Car s'il convient d'insister sur l'importance du contrôle personnifié (celui du dirigeant) dans la définition de la petite entreprise, il faut aussi laisser la porte ouverte, conformément à la vision du système productif développée par Richardson (1972), à diverses collaborations plus ou moins équilibrées avec d'autres entreprises et donc à un contrôle élargi ou partagé (Guilhon, 1998).

Ainsi, si la plupart des petites entreprises peuvent être analysées avec profit en termes de proximité (Torrès, 1998, 2003)1, on aurait tort de voir dans cette

\footnotetext{
${ }^{1}$ Torrès avance que le mécanisme théorique fondant la conception classique de la PME et délimitant le cadre de validité du paradigme de la spécificité des modes de gestion de la PME est la proximité. Référence est faite ici à la proximité hiérarchique (centralisation et personnalisation de la gestion, ligne hiérarchique réduite, coordination des membres de l'entreprise par supervision directe ou ajustement mutuel), fonctionnelle (omniprésence et polyvalence du dirigeant, faiblesse voire inexistence de la séparation des tâches et des fonctions, forte imbrication entre les décisions stratégiques, administratives et opérationnelles) et temporelle (préférence pour le court terme, attitude plus réactive qu'anticipative, quasi-absence de planification notamment en matière de formation), ainsi qu'au capital de proximité (capital souvent constitué de fonds personnels et d'origine familiale, préférence pour les réseaux régionaux de financement, personnalisation de la relation dirigeant - banquier, importance du capital relationnel) ou bien encore au fort ancrage local de nombreuses PME.
}

dernière le mécanisme qui fonde la spécificité de la petite entreprise. Une entreprise de petite taille qui ne s'appuie pas sur la famille du dirigeant, qui recourt à une certaine formalisation (de la stratégie, du système d'information et/ou de la formation) et qui ne s'adresse pas à un marché local peut, dans bien des cas, être légitimement considérée comme une petite entreprise à part entière. Des modes de gestion introduisant plus de distanciation au sein de l'entreprise comme entre celle-ci et son environnement ne « dénaturent » pas la petite entreprise tant que le dirigeant est à l'origine de ce mode de fonctionnement et ne perd pas le contrôle de son affaire. Au total, la caractérisation provisoire de la petite entreprise comme quasi-organisation autorise une mise en cohérence des similitudes observées. Elle échappe ainsi au modèle de l'hyperfirme (axée sur la poursuite des économies d'échelle), qui pousse l'observateur de la vie économique à concevoir la petite entreprise en termes de manques et ne permet pas de comprendre comment des unités parviennent à prospérer en restant, le plus souvent sciemment, en deçà de la frontière «optimale » suggérée par l'économie industrielle. On est également amené à positionner la petite entreprise dans la dynamique de l'organisation sociale et industrielle à laquelle elle participe. Dès lors, un même format d'entreprise peut être organisé et mobilisé de plusieurs manières, en fonction des choix des acteurs en présence.

\section{Trois manières de s'appuyer sur des réseaux}

Pourquoi ce regain d'intérêt pour les PME voire, depuis peu, pour les TPE (très petites entreprises)? Probablement que le format proposé par la petite entreprise est gage d'efficacité dans certaines conditions de marché actuelles. Nous parlons d'un format dans la mesure où il est bien question d'un modèle qui définit les règles à observer pour la dimension et la disposition des unités économiques: entreprises mais aussi établissements, ateliers, services, groupes... Par analogie avec les systèmes informatiques, nous pourrions dire que le système productif tend à être formaté en fonction du prisme de la petite entreprise, l'engouement pour ce format étant entretenu aussi bien par le desserrement de certaines contraintes technologiques et sociales (production 
« au plus juste », recherche tous azimuts de flexibilité, développement de l'échange de données informatisées, responsabilisation des travailleurs, meilleure protection des indépendants, etc.), que par l'essor des relations de service. Tout cela contribue à ouvrir ou élargir des espaces de développement aux petites entreprises. Mais, si ces dernières sont globalement pertinentes dans le système économique actuel, elles peuvent l'être de bien des manières. Nous proposons de distinguer trois manifestations majeures du format petite entreprise. Nous évoquerons tour à tour les petites entreprises traditionnelles, managériales et néolibérales, et indiquerons dans quel cadre chacune de ces manifestations est préférentiellement mobilisée.

Petites entreprises traditionnelles: ancrage local et réseaux affinitaires

Les petites entreprises que nous qualifions de traditionnelles se veulent avant tout indépendantes et sont gérées « en bon père de famille». Elles ont vocation à durer, leurs dirigeants étant particulièrement attachés à la pérennité de l'affaire et espérant, dans bon nombre de cas, la transmettre à un ou plusieurs membres de leur entourage. Dégager un revenu décent pour le chef d'entreprise et sa famille, tout en faisant progresser au fil des années la valeur patrimoniale des actifs, telle est la fonction économique de l'entreprise. Ces entreprises suivent généralement des stratégies qualifiées d'émergentes qui souvent se caractérisent, du point de vue de l'observateur extérieur, par... une absence de stratégie. En réalité, tout est fait pour pacifier, neutraliser la concurrence en se partageant, entre petites entreprises traditionnelles, les « rentes» d'un territoire commun. Ces firmes sont à la fois très poreuses par rapport à leur environnement professionnel local (la clientèle est conçue comme une collection de personnes et non comme l'agrégation de demandeurs indifférenciés; les concurrents sont souvent considérés comme des collègues, etc.) et très étanches à l'égard de l'environnement dit parfois «de soutien». D'où une méfiance évidente face aux diverses administrations, aux organismes professionnels, aux offreurs de formation... bref, face à tous ceux qui, souvent, sont considérés par le dirigeant comme des « intrus », des «perturbateurs » qui plus est enclins à taxer son entreprise de passéiste.
Mais pourquoi existe-t-il encore de telles firmes présumées archaïques? Deux explications plus complémentaires qu'alternatives peuvent être avancées. La première, sans doute la plus répandue et la plus négative, consiste à remarquer qu'à côté des grandes entreprises qui déterminent la dynamique du capitalisme, il a de tout temps existé une place, en marge des firmes internationales et des PME innovantes et dynamiques, pour des entreprises relativement peu performantes, fuyant la concurrence et dont les dirigeants se contentent de maigres revenus. Cette place est évidemment d'autant plus grande que la dynamique du salariat est enrayée, un certain nombre d'individus étant alors contraints de créer leur propre emploi. Cette explication privilégiant l'impact du chômage de masse appelle bien sûr des réponses en termes de formation au moment de la création d'entreprise et, idéalement, lors de la phase de démarrage. Une deuxième raison fait référence à la persistance de systèmes socioproductifs qui ont su, sur longue période, maintenir leur cohérence ainsi que leur spécificité. L'artisanat et le petit commerce ainsi que des pans entiers de certains secteurs d'activité (dans l'hôtellerie-restauration ou l'agroalimentaire par exemple) se rattachent encore à un ensemble de valeurs individuelles et sociales traditionnelles: valeurs familiales, volonté farouche d'indépendance, recherche de pérennisation de l'entreprise, sentiment d'appartenance à un métier, attachement à la hiérarchie maître - compagnon - apprenti, etc. Ici, les pratiques formatives sont certes plus nombreuses mais elles empruntent des voies particulières (l'apprentissage par exemple) et souvent informelles.

\section{Petites entreprises managériales: instrumentalisation gestionnaire et réseaux hiérarchiques}

Les petites entreprises managériales obéissent essentiellement à une rationalité économique. Elles doivent avant tout assurer une rémunération élevée à leurs dirigeants, tout en leur apportant prestige et sécurité. Pour ce faire, leurs dirigeants renoncent à l'indépendance absolue revendiquée par l'artisan mais s'appuient sur une «technologie invisible» (l'instrumentalisation gestionnaire) et, éventuellement, une infrastructure qui a priori leur permettent d'espérer des revenus supérieurs. Les petites entreprises managériales sont donc 
les plus concernées par les réseaux formels et hiérarchiques : sous-traitance, franchise, concession... Cela ne les dispense évidemment pas de participer, à l'occasion, à des réseaux informels territorialisés mais, à l'évidence, leur mode de fonctionnement et leur cohérence ne reposent pas uniquement sur la proximité. Nous sommes plutôt en présence d'un régime d'action à distance (Bessy, Boisard et EymardDuvernay, 1995 ; Lagarenne et Marchal, 1995) ou, plus souvent encore, d'un régime d'action intermédiaire ou composite (Thévenot, 1993) que l'on pourrait qualifier de "proximité distanciée » (Trouvé, 1997)2. Bien entendu, cela les conduit à adopter un fonctionnement largement calqué sur le modèle de la grande entreprise. Le rôle de chacun, par exemple, est assez clairement identifié et s'inscrit dans une division interne du travail qui tranche avec la division sociale du travail de type quasi familial des petites entreprises traditionnelles. L'instrumentation gestionnaire qui a cours dans ces firmes favorise la mise en œuvre de stratégies (de formation notamment) et s'appuient sur la construction et le suivi de divers indicateurs.

Ces entreprises sont de plus en plus nombreuses, non seulement parce que le système éducatif tend à générer des flux importants de managers mais aussi parce que cette manifestation du format petite entreprise est fortement sollicitée, mobilisée par le capitalisme moderne, prioritairement dans le cadre de groupes (Debray et Leyronas, 1998 ; Mahé de Boislandelle, 1998). On peut schématiquement distinguer (Ardenti et Vrain, 1998 ; Bentabet, Michun et Trouvé, 1999) :

- les petites entreprises « intégrées » qui appartiennent à des groupes ou à des réseaux de franchise, de concession ou de sous-traitance (commerce, réparation automobile, hôtellerie-restauration, etc.): le «partenaire » dominant est à l'origine de l'instrumentation gestionnaire qui, du coup, est imitée, induite ou carrément imposée ;

- les petites entreprises « modernes » qui, d'emblée, ont été imaginées et organisées par leur créateur

\footnotetext{
${ }^{2}$ Les notions de régime d'action de proximité à distance et de proximité distanciée s'inspirent des travaux du Centre d'études de l'emploi sur les recrutements. Cependant, la perspective est ici plus large puisqu'il s'agit de rendre compte de la cohérence des petites entreprises tant interne (division du travail, rapports dirigeant/salariés, etc.) qu'externe (relations avec l'environnement). Elle recoupe les travaux plus généraux de Boltanski et Thévenot (1987) sur les économies de la grandeur, d'Eymard-Duvernay (1987) sur les modèles d'entreprises ou de Salais et Storper (1993) sur les mondes de production.
}

selon des modalités managériales (montage d'une société plutôt que d'une entreprise individuelle, ouverture du capital, stratégie planifiée, formalisation des pratiques, etc.) ;

- les petites entreprises « modernisées » qui, auparavant, relevaient du modèle traditionnel et qui - sous la pression de certains événements (achat d'équipement, développement d'un marché à l'exportation, arrivée d'un nouveau salarié hautement qualifié, etc.), d'une réorientation stratégique du dirigeant ou, plus souvent, d'un changement de dirigeant - basculent vers des modes de fonctionnement plus gestionnaires.

Signalons que les qualificatifs «modernes» ou «modernisées» utilisés par les auteurs précités ne doivent pas conduire à considérer que le modèle managérial est en toutes occasions préférable et a par conséquent vocation à supplanter tous les autres. Le profil du dirigeant (Marchesnay, 1991), le positionnement de la firme dans son espace concurrentiel (Fernandez, Picory et Rowe, 1996), la combinaison productive retenue (Bentabet, Michun et Trouvé, 1999) mais aussi les caractéristiques de l'offre de services en gestion (Marchesnay, 1992) n'imposent pas nécessairement comme meilleure solution le recours à des procédures managériales. Sans compter que les processus cognitifs entrepreneuriaux réellement en œuvre dans les petites entreprises sont généralement trop complexes pour s'accommoder d'une simple adaptation de procédures ayant fait leur preuve dans les grandes entreprises (Marchesnay, 1995).

Petites entreprises néolibérales: marché professionnel du travail et réseaux de projet

Enfin, à côté des petites entreprises traditionnelles et managériales, on trouve quelques petites entreprises néolibérales. Elles se rapprochent le plus du modèle de l'hypofirme développé par Marchesnay (1993). Elles ont effectivement pour fonction-objectif de maximiser la valeur sous contrainte d'une taille plafond (fixée par le dirigeant) à ne dépasser à aucun prix ; ceci les distingue nettement des deux autres types.

D'abord, des petites entreprises traditionnelles qui, portées par une logique patrimoniale, ne sont pas $a$ priori opposées à une progression maîtrisée de la taille, pourvu que cette augmentation de la taille ne se 
fasse pas au détriment de la pérennité et de l'indépendance. La logique d'accumulation peut donc conduire le dirigeant à franchir le seuil des dix ou, beaucoup plus rarement, celui des cinquante salariés. Cela reste envisageable tant qu'il parvient à conserver le contrôle de son affaire et à maintenir un fonctionnement axé sur la proximité, par exemple en montant un groupe de petites entreprises dirigées par lui-même ou des membres de son entourage (hypogroupe);

Ensuite, des petites entreprises managériales dont les dirigeants, au profil de manager, sont intéressés avant tout par les flux de revenus que dégage leur affaire et leur taux de croissance. Ils n'ont, en principe, aucun scrupule à franchir tel ou tel seuil, d'autant qu'ils ont souvent des compétences avérées en matière d'organisation. Et si leur firme n'a généralement pas vocation à devenir «moyenne», c'est qu'une taille modeste demeure un atout certain pour les activités dans lesquelles ils se lancent préférentiellement (les services aux entreprises par exemple), sans compter qu'une telle taille autorise, au besoin, un désengagement rapide et à moindre frais.

Seule la petite entreprise néolibérale peut être analysée comme un centre de décision et de contractualisation dont la taille est volontairement limitée au strict minimum. Les transactions « externes » sont ici très nettement privilégiées, sans pour autant être aveuglément confiées au marché. Au contraire, un effort particulier est fait pour aménager puis entretenir un espace de transaction à partir duquel la firme peut développer sa stratégie de spécialisation. On observe de telles pratiques essentiellement dans les services (organismes de formation, entreprises de traduction, etc.), ainsi que dans certaines professions de la filière textile-habillement (Courault et Parat, 1998). D'autres exemples sont suggérés par Greffe (1999) dans le cadre de son analyse de l'emploi culturel. De plus en plus d'intervenants (des acteurs par exemple), nous dit-il, peuvent être conçus comme des « quasi-firmes ». En effet, ils se comportent au quotidien en travailleurs indépendants gérant eux-mêmes leur carrière, leur perfectionnement et, souvent, leur diversification - tout en participant régulièrement à des «entreprises » collectives autour de projets particuliers. Cette notion de projet est centrale pour la petite entreprise néolibérale. Le projet définit en effet l'étendue de la contractualisation (bien au-delà du «noyau dur», du «nœud» des salariés permanents) et sa durabilité. Il fixe les compétences à mobiliser et les synergies à organiser.

Ce mode de fonctionnement limite de façon draconienne les coûts d'acquisition, de conservation et de développement des ressources humaines, dans la mesure où l'essentiel de ces coûts sont externalisés, répercutés sur des individus «autonomes». Encore faut-il que le marché du travail suive, que les individus acceptent cette mobilité et ce fonctionnement par projet et que la petite entreprise néolibérale se donne les moyens de créer et d'entretenir un tissu dense de relations dans lequel elle peut puiser. C'est dire que les petites entreprises néolibérales ne peuvent se développer tous azimuts. Leur développement exige des conditions permissives (structuration forte du secteur ou de la profession, qualifications transférables, règles spécifiques de protection des travailleurs, etc.) particulières, sensiblement éloignées du salariat classique auquel la plupart des individus demeurent à l'évidence attachés. Sans parler de l'indispensable bonne réputation de la firme et de chacun des membres de son réseau "externe », réputation qui ne va pas de soi et qui passe souvent par des relations personnalisées et des collaborations répétées.

On retiendra, au final, que si les petites entreprises ne peuvent se concevoir indépendamment du milieu social, économique, éducatif dans lequel elles évoluent, cela n'est pas toujours synonyme d'ancrage local fort, pour ne pas dire exclusif.

\section{PETITES ENTREPRISES TRADITIONNELLES ET « RÉSEAUX APPRENANTS »}

Les partenaires sociaux qui, à l'heure actuelle, dénoncent tous à leur manière la persistance des inégalités face à la FPC ne peuvent se permettre d'ignorer les spécificités et la diversité du milieu des petites entreprises.

Certes, dans le cas particulier des petites entreprises managériales, il est à la rigueur possible d'en rester à une simple adaptation des «produits» existants et 
ayant fait leur preuve pour de moyennes ou grandes entreprises. Ici, un marché de la formation existe déjà et ne demande qu'à s'étoffer via notamment l'intervention d'intermédiaires financiers tels que les OPCA (Organismes paritaires collecteurs agréés).

Mais déjà, dans le cas des petites entreprises néolibérales, il devient difficile de se contenter d'une simple transposition car la formation continue y est d'abord et avant tout l'affaire de professionnels amenés à gérer leur mobilité interentreprise au gré des projets qui s'offrent à eux. Une réelle individualisation de la formation au niveau des contenus pédagogiques est à imaginer. Il conviendrait également de donner corps au droit individuel à la formation (référence au DIF qui se met en place dans le cadre de la réforme en cours de la FPC), penser sa transférabilité d'une entreprise à l'autre et faire appliquer une réelle mutualisation financière au niveau des fonds collectés par les OPCA. En effet, malgré la réforme du système de FPC, l'employeur demeure l'initiateur (et le décideur) principal voire, dans bien des cas, unique en matière de formation. En outre, les entreprises les plus structurées et les plus formatrices, maîtresses de leurs actions et dépenses, tendent à « assécher » les OPCA constamment confrontés à une contrainte financière forte. L'individu isolé et amené à changer fréquemment d'employeur a, dans ce contexte, peu de chances de bénéficier d'un soutien financier et d'un accompagnement significatifs en matière de formation.

Ce sont surtout les petites entreprises traditionnelles qui posent problème. C'est donc sur elles que nous allons désormais focaliser notre attention. Nous insisterons tout d'abord sur le perfectionnement des dirigeants, préalable indispensable à l'introduction de la FPC dans ces entreprises. Nous évoquerons ensuite l'improbable «dialogue social» dans les petites entreprises traditionnelles, dialogue pourtant devenu incontournable dans le cadre de la réforme de la FPC. À chaque fois, l'approche territoriale et les réseaux apporteront des éléments de réponse (Bentabet et Michun, 2002, 2003).

\section{Le territoire comme espace d'intelligibilité}

Le territoire constitue un facteur important de l'identité des petites entreprises : ce n'est pas un espace - support indifférencié et sans effet sur le dirigeant et sa firme. Il offre un horizon de référence, un cadre de sécurité relative et un «espace de lecture » (Goujet, Pastorello et Silvestre, 1994) à partir duquel l'entreprise peut développer ses diverses activités. Dans le meilleur des cas, la réduction d'incertitude qui en découle, en minorant les coûts d'information des entrepreneurs, autorise une diminution des coûts de transaction et, finalement, une interpénétration plus ou moins poussée des différentes organisations locales. Cette interpénétration a d'ailleurs toujours été considérée comme le secret du succès des systèmes productifs localisés, que ceux-ci soient le produit d'une histoire spécifique ou qu'ils se réfèrent plus explicitement au volontarisme politicoéconomique, comme dans le cas des technopoles (RERU, 1991 ; 1993 ; 1999).

Ces systèmes productifs localisés ont certes des particularités qui tiennent à la création et à l'évolution permanente d'un certain nombre de normes, de procédures participant à la rationalité des agents économiques locaux et assurant au système luimême sa stabilité, sa continuité et sa cohérence. Mais cela n'empêche pas les petites entreprises traditionnelles extérieures à ces zones singulières de procéder d'un mode de coordination territorialisé, ce qui peut conduire à rejeter à la périphérie tout ce qui ne relève pas du « face-à-face », de la proximité marchande et de la relation de confiance, comme les simples «contacts» institutionnels qui sont vécus le plus souvent, comme « procéduriers » ou déconnectés des réalités de la petite entreprise. Autrement dit, si la rationalité des dirigeants de petites entreprises n'est pas plus limitée que celle des dirigeants de firmes imposantes, elle est dans une large mesure plus contextuelle. Le sort des unités de faible dimension dépend étroitement des caractéristiques de l'environnement immédiat. Pour les petites entreprises traditionnelles, «la proximité est un mécanisme de hiérarchisation qui créée les conditions nécessaires à l'action et à la réflexion dans une organisation centralisée, faiblement spécialisée, dotée de système d'information interne et externe simples et privilégiant des stratégies intuitives et peu formalisées 》 (Torres, 2003, p. 119).

Vouloir aider les entreprises confrontées à un environnement particulièrement turbulent et complexe afin qu'elles se modernisent, qu'elles intègrent 


\section{Encadré \\ Par delà les réformes, la délimitation de la formation professionnelle continue évolue peu}

La norme et le "modèle " de la grande entreprise se sont cristallisés dans la définition officielle de la formation continue telle qu'elle apparaît dans la loi de juillet 1971. Des règles financières - utilisées pour déterminer ce qui est imputable et ce qui ne l'est pas - et sociales (favorisant, par exemple, la négociation du plan de formation dans le cadre du comité d'entreprise) y sont fixées en s'appuyant sur quatre critères particulièrement restrictifs : la notion d'action programmée, la nécessité d'une progression pédagogique, la présence d'un formateur, l'extériorité des locaux de formation par rapport à la production (Dayan et al., 1986). Ces critères, une fois repris par l'appareil statistique, notamment au travers de la déclaration fiscale 24.83, ne peuvent, bien évidemment, que minorer fortement la contribution des petites entreprises à la formation professionnelle continue (FPC). Que n'a-ton pu dire sur le " retard " des petites entreprises en matière de FPC, sur leur " amateurisme», sans jamais envisager autre chose que la transposition de ce que l'on sait faire pour la grande entreprise !

Notons que la réforme de la FPC, intervenue en 2004, ne revient guère sur cette délimitation très restrictive de la formation continue. "Le compromis auquel sont parvenus les partenaires sociaux après un an de négociation ne remet pas en cause les grandes lignes du cadre institutionnel de la formation professionnelle continue tel qu'il avait été établi par l'accord national interprofessionnel de 1970 et la loi de 1971 : le plan de formation, assorti d'une obligation de dépenses basée sur la masse salariale, et le congé individuel de formation financé par une contribution des entreprises à des fonds mutualisés, demeurent les deux piliers du système. L'accord national interprofessionnel du 20 septembre, loin de remettre en cause ce socle, considéré à juste titre comme une singularité française, s'inscrit délibérément dans son prolongement. De même le rôle conféré aux branches professionnelles à travers la mutualisation des fonds consacrés aux formations en alternance et au plan de formation, se trouve confirmé par l'accord et sa transposition législative » (Merle, 2004).

Cette focalisation sur la planification de la formation (*) (peut-être souhaitable à moyen terme mais inconcevable dans l'immédiat pour nombre de petites entreprises) couplée au pouvoir accru accordé aux branches professionnelles ne peut que renforcer la vision homogène et univoque de l'investissement formation. De même, le Droit individuel à la formation (DIF) - qui est censé permettre au salarié de se constituer un contingent d'heures de formation à utiliser à son initiative après accord de l'employeur sur le choix de l'action - fait l'impasse sur les conditions de travail dans les petites entreprises et les relations directes (sans médiation syndicale par exemple) entre le patron et ses salariés qui y ont cours.

(*) : Le plan de formation est, en outre, rendu plus complexe (et artificiel ?) par l'obligation d'y faire apparaitre clairement trois catégories d'actions de formation: les actions d'adaptation au poste de travail; les actions de formation liées à l'évolution des emplois et au maintien dans l'emploi ; les actions de formation liées au développement des compétences.

pleinement les nouveaux facteurs de compétitivité et qu'elles recourent à la formation continue n'est alors possible qu'après avoir établi une relation personnalisée fondée sur la confiance avec leurs dirigeants. Ce contact permanent, non pas entre une entreprise et une institution (un établissement, un organisme, une association...) mais entre deux ou plusieurs personnes qui apprennent à se connaître, permet de modifier positivement les représentations des uns et des autres. Ainsi, bon nombre d'efforts indiscutables en matière de formation initiale ou continue sont-ils insuffisamment récompensés, faute de relations véritables avec les dirigeants de petites entreprises. Avant de les questionner sur leurs attentes (pour obtenir le plus souvent des réponses biaisées et non opérationnelles) ou même de leur demander d'accueillir des 
stagiaires ou des personnes en alternance, il conviendrait d'abord de chercher à comprendre, au cas par cas, leurs cadres de référence, leurs façons de penser et d'agir, leurs projets et leurs contraintes (ne seraitce qu'en partant de leurs pratiques de recrutement et de formation « sur le tas »).

C'est tout l'intérêt de l'engagement de développement de la formation (EDDF) collectif dont ont bénéficié les entreprises du Club Alliance Métaux pour le Développement des Industries du Biterrois (Camdib), en Languedoc-Roussillon ${ }^{3}$. Cette association d'industriels, créée en 1996, recouvre 31 établissements (appartenant à 24 entreprises, dont 15 comptent moins de 20 salariés et 4 plus de 50 à 280 salariés) de la filière «travaux des métaux » qui emploient environ 900 salariés et réalisent un chiffre d'affaires de plus de 100 millions d'euros. Devant le constat d'une trop grande dépendance à l'égard de donneurs d'ordre locaux et d'un besoin crucial de formations spécifiques, les industriels ont décidé de constituer un réseau, Camdib, puis d'initier leur propre programme de formation. Trois types de formations ont été mises en œuvre : des formations internes, en partie informelles et propres à chaque entreprise, des formations externes auprès d'organismes de formation et enfin, des formations interentreprises, au sein de Camdib, afin de permettre des échanges de pratiques professionnelles, éventuellement complétés par des apports d'experts. Le plan de formation a été structuré et formalisé autour des problématiques communes des entreprises du territoire, après de multiples discussions entre les dirigeants. Les pouvoirs publics (DRTEFP - Direction régionale du Travail, de l'Emploi et de la Formation professionnelle, FSE - Fonds social européen, DRIRE - Direction régionale de l'Industrie, de la Recherche, et de l'Environnement) secondés par l'AGEFOS-PME, ont assuré l'essentiel $\mathrm{du}$ financement et se sont largement adaptés aux modes de fonctionnement des entreprises de ce club

\footnotetext{
${ }^{3}$ L'association voit le jour fin 1996, avec pour objectif de développer l'action commerciale dans quatre métiers : la tôlerie-emboutissage, le traitement de surface, les produits du bâtiment et les ouvrages hydrauliques. Vingt-deux entreprises, identifiées lors d'une étude préalable et représentant presque 81 millions d'euros de chiffre d'affaires et 820 emplois, acceptent de participer à la mise en place d'actions structurantes : échanges d'expériences, achat en commun d'outils productifs, mise en place d'actions de formation, etc.
}

de dirigeants ${ }^{4}$. À titre d'exemple, les entreprises ont pu largement bénéficier de formations internes prenant appui sur l'outil de travail habituel des salariés. De même, il a été possible de former des salariés déjà expérimentés afin, qu'à leur tour, ils deviennent « formateurs » au sein de leur entreprise.

\section{Les réseaux : s'ouvrir à la formation, tout en restant le patron}

Il n'y a pas de formation continue en petite entreprise si le dirigeant n'est pas convaincu, non seulement de son utilité et de son efficacité à court terme mais aussi de sa compatibilité avec le fonctionnement quasi familial de son affaire. Il ne suffit donc pas de régler les problèmes de coût, de disponibilité et d'adaptation des contenus de formation. Il s'agit d'abord de régler la question du perfectionnement des chefs d'entreprise : c'est la clé de l'introduction, puis du développement de la FPC dans ces quasi-organisations. Autrement dit, une approche économique du «marché de la formation continue» des dirigeants et salariés de petites entreprises est insuffisante. Il est vain de compter sur un mécanisme « spontané » d'ajustement entre des offres et des demandes. Il est nécessaire de mettre en perspective le processus de perfectionnement en admettant la complexité des modes de pensée et d'action des dirigeants.

Compte tenu de son attachement à son autorité légitime et de son identification à son entreprise, le dirigeant peut se sentir déstabilisé et remis en question dès lors que son perfectionnement - et donc ses «manques »- sont exposés dans son entreprise et plus encore auprès de formateurs extérieurs En revanche, le réseau construit un système familier de relations acceptées et largement maîtrisées par le dirigeant, qui favorise la mise en œuvre de démarches informelles d'appropriation de connaissances. Par leur inscription dans des réseaux, les dirigeants parviennent à «tenir leur rang» tout en mettant à profit des relations interindividuelles affinitaires pour

${ }^{4} \mathrm{La}$ DRTEFP et le FSE ont été à l'origine de l'EDDF et en ont défini le cadre et les limites avec les entreprises membres de Camdib ; l'AGEFOS-PME, en tant qu'organisme paritaire collecteur agréé, a contribué au cofinancement des actions de formation (en plus des contributions des entreprises et de l'apport du FSE); enfin, la DRIRE a accompagné la modernisation des entreprises, notamment en leur permettant de se rapprocher, en termes de compétences, des standards attendus par les principaux donneurs d'ordres. 
résoudre tel ou tel problème particulier. Les citations suivantes émanant de patrons membres de Camdib en témoignent :

"On est dans un cadre, on réfléchit avec les autres patrons ; ça change car quand on est chef d'entreprise, on est tout seul. On se soutient entre nous. Camdib, c'est une petite famille maintenant ».

«On rencontre des gens qui ont les mêmes problèmes que nous. On se rencontre, on collabore et on élabore des stratégies ensemble. C'est réconfortant car un chef d'entreprise est souvent tout seul ».

«Le réseau Camdib a permis des échanges fabuleux. On n'est pas forcément du même métier mais on a des entreprises complémentaires. Beaucoup d'entreprises de Camdib travaillent les unes avec les autres. On a une multitude de contacts qui nous permettent d'avoir des échanges sur les difficultés, les personnels, les formations ».

Parfois, le réseau permet de dépasser le simple stade de la logique de résolution de problèmes particuliers. Tel est le cas lorsque des dirigeants s'engagent consciemment dans un questionnement collectif autour de problèmes communs et ouvrent leur réseau à des experts. Ainsi, au sein de Camdib, des études de marché, des démarches de gestion prévisionnelle des emplois et des compétences (GPEC) ainsi que des coopérations technologiques avec des centres de recherches ont-elles été initiées.

On est ici véritablement dans le champ de la formation car le processus d'apprentissage est volontaire, conscientisé a priori et associé à des solutions génériques. La formation des salariés devient alors envisageable. Ainsi, sur la période 2001-2003, 1728 actions de formation ont été menées et 50933 heures de formation ont été dispensées dans le cadre de l'EDDF dont ont bénéficié les entreprises de Camdib (de 2001 à 2003, 526 personnes sur un effectif total d'environ 900 salariés ont bénéficié de l'EDDF). Les entreprises de moins de 20 salariés ont ainsi pu former une large partie de leur ressource humaine, en particulier leurs dirigeants non salariés (33 dirigeants ont profité de cette opportunité) et leurs cadres (153 cadres appartenant à des entreprises de moins de 20 salariés ont bénéficié de formations dans le cadre de l'EDDF, ce qui représente presque la moitié des cadres formés) mais aussi leurs ouvriers non qualifiés (au nombre de 67 sur les 139 ouvriers non qualifiés formés) ou qualifiés (175 sur 644).

\section{Réseaux d'entreprises : le dialogue social délaissé}

Un problème demeure. Dans les petites entreprises, le dialogue social est fréquemment inorganisé, informel et déséquilibré en faveur de l'employeur. D'où la difficulté des petites entreprises à adopter les préceptes de la négociation collective, c'est-à-dire à s'ouvrir sur des formes de régulations sociales construites à l'extérieur de leur enceinte. Mais cela ne signifie pas l'absence d'échanges dans les rapports au travail entre salariés et entre salariés et employeurs. Ce n'est pas tant l'existence d'échanges qui pose problème (ils sont en fait très nombreux dans ces quasi-organisations) que leur caractère complexe (et non collectif) car informel, personnalisé et potentiellement instable. Comment faire pour formaliser et réguler les rapports sociaux face à un "collectif de travail » peu structuré ? Comment élever le degré de perméabilité des petites entreprises aux normes externes, comme les conventions de branche?

Une solution se dessine, mais elle demeure en marge de tout ce qui est associé par les dirigeants à l'institution et à l'administration. Il s'agit, là aussi, des réseaux conçus ici comme des points de passage obligés des règles sociales. En effet, l'inscription dans un réseau, même informel, implique l'acceptation de ses règles économiques et sociales, constitutives d'identités collectives propres. Il conviendrait donc de connaître et d'utiliser ces innombrables réseaux (clubs et associations de dirigeants, groupements d'employeurs, organismes professionnels mais aussi réseaux de franchise et autres réseaux associés aux nouvelles formes de sous-traitance) ainsi que les conseillers et prescripteurs, tels les experts-comptables, souvent au croisement de ces réseaux. Le caractère plus normatif qu'impératif des règles circulant par leur intermédiaire semble mieux répondre à la nature des relations internes dans les petites entreprises. Reste que les salariés sont souvent peu impliqués dans ce type de réseaux et y voient un simple «club de patrons ». Les pouvoirs publics, forts de leurs engagements financiers décisifs dans ce type de réseaux, ne devraient-ils pas 
désormais exiger des dirigeants des avancées en ce domaine ? Sans doute les engagements de développement de l'emploi et des compétences (EDEC) résultant de la fusion des contrats d'études prospectives (CEP) et des engagements de développement de la formation (EDDF) y contribueront-ils.

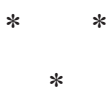

Afin de tenter de résorber les inégalités d'accès à la formation, il convient de considérer que la plupart des petites entreprises ne sont pas de grandes entreprises en miniature. Ainsi, il est nécessaire d'innover et d'accepter la diversité de ce milieu. Autrement dit, il s'agit en permanence de revenir sur la spécificité de ces entreprises dites à « taille humaine», celle d'une firme par certains aspects « incomplète» (eu égard au modèle de la grande organisation) et, partant, profondément tributaire de son environnement comme des individus qui la composent (le dirigeant mais aussi les salariés, y compris les jeunes débutants « déversés » en masse par le système éducatif et sommés de se construire une expérience professionnelle).

Dès lors, la proximité peut servir de levier pour développer la formation continue dans les petites entreprises traditionnelles, tant attachées aux réseaux affinitaires. Dans certains cas, la proximité géographique peut en effet légitimement être conçue comme un intermédiaire social susceptible de réduire l'incertitude et d'orienter les comportements des dirigeants de petites entreprises. Confrontés à un environnement économique et technologique mouvant, instable, souvent imprévisible, ces derniers trouvent parfois dans la localité un espace de relative stabilité permettant d'envisager des relations s'inscrivant dans le long terme et reposant sur une confiance réciproque. Reste que la coexistence d'entreprises privilégiant, chacune de leur côté, les relations de proximité ne suffit pas, à elle seule, à générer un territoire spécifique (i.e. présentant pour les directions d'entreprise comme pour les salariés des avantages décisifs par rapport à d'autres espaces plus indifférenciés). Souvent, il n'y a pas assez de synergies; 1'articulation petites entreprises/développement local fonctionne mal. Ce scenario pessimiste est d'autant plus fréquent en France que le poids des branches professionnelles y demeure écrasant et relègue au second plan les territoires. Dès lors, l'approche de la formation demeure très centralisée, le territoire n'étant qu'un espace support sur lequel on décline des priorités et des outils établis selon une logique verticale. Si cela peut s'accorder avec les pratiques des petites entreprises managériales, il est clair que seul le «développement par le bas", en appui sur les réseaux entrepreneuriaux, peut contribuer à combler les insuffisances des petites entreprises traditionnelles en matière de formation continue.

\section{Bibliographie}

Ardenti R. et Vrain Ph. (1998), Relations interentreprises, profils des dirigeants et gestion de l'emploi dans les PMI, Convention d'étude entre la DARES et le CEE, 98/09, Paris, CEE.

Bentabet E., Michun S. et Trouvé Ph. (1999), Gestion des hommes et formation dans les très petites entreprises, Marseille, Céreq, collection «Études », $\mathrm{n}^{\circ} 72$.

Bentabet E. et Michun S. (2002), De l'apport des organismes collecteurs au dynamisme du marché de la formation : la difficile approche des petites entreprises, Marseille, Céreq, Collection « Document », n 166.

Bentabet E. et Michun S. (2003), «Petites entreprises et réseaux : quelle intermédiation pour la formation continue?», Formation Emploi, n ${ }^{\circ} 84$, octobredécembre, Paris, La Documentation française, pp. 6780.

Bessy C., Boisard P. et Aymard-Duvernay F. (1995), Jugements à distance et relations de proximité dans 
les recrutements, CEE, Rapport de la convention CEE/ANPE sur les intermédiaires du recrutement.

Boltanski L. et Thévenot L. (1987), « Les économies de la grandeur", Cahiers du Centre d'Études de l'Emploi, n 31, Paris, PUF.

Courault B. et Parat E. (1998), «PME et emploi : l'industrie de l'habillement à Roanne et à Cholet ", La Lettre du CEE, $\mathrm{n}^{\circ}$ 54, octobre.

Dayan J.-L., Géhin J.-P., Verdier É. (1986), « La formation continue dans les petites et moyennes entreprises: spécificités et paradoxes ", Formation Emploi, n ${ }^{\circ}$ 16, octobre -décembre, pp. 7-36.

Debray C. et Leyronas C. (1998), «Le réseau et l'hypogroupe: de nouvelles stratégies organisationnelles en PME », in Torrès O. (Dir.), De nouvelles approches pour comprendre les PME, pp. 83-93.

Eymard-Duvernay F. (1987), «Les entreprises et leurs modèles », Cahiers du Centre d'Études de l'Emploi, $\mathrm{n}^{\circ}$ 30, Paris, PUF, pp. 5-12.

Fernandez V., Picory C. et Rowe F. (1996), « Outils de gestion et espaces concurrentiels des PME », Revue Internationale PME, Vol. 9, n 1, pp. 79-102.

Goujet R., Pastorello M.-H, Silvestre H. (1994), «Eléments de recherche sur la relation des PMI avec leur environnement. Cadre d'étude : les systèmes productifs locaux ", Communication à la $39^{e}$ Conférence Mondiale de l'ICSB : Les PME/PMI et leur contribution au développement régional et international, pp. 269-285.

Greffe X. (1999), L'emploi culturel à l'âge du numérique, Paris, Anthropos.

Guilhon A. (1998), «Vers une nouvelle définition de la PME à partir du concept de contrôlabilité », in Torrès O., PME : de nouvelles approches, pp. 55-67.

Lagarenne C. et Marchal E. (1995), « Recrutements et recherche d'emploi », La lettre du CEE, $\mathrm{n}^{\circ} 38$, juin.

Mahé de Boislandelle H. (1998), "GRH en PME. Universalité et contingences : essai de théorisation », Revue Internationale PME, Vol. 11, n²/3, pp. 11-30.

Marchesnay M. (1991), «La PME: une gestion spécifique ? ", Économie Rurale, n 206, novembre/ décembre, pp. 11-17.
Marchesnay M. (1992), « L'offre de services en gestion à la petite entreprise », in Labourdette A. (Dir.), Mélanges en l'honneur de Jean-Guy Mérigot, Paris, Économica, pp. 445-466.

Marchesnay M. (1993), Management stratégique, Paris, Eyrolles.

Marchesnay M. (1995), Information, risque et système de gestion de l'hypofirme, Deuxième congrès international francophone de la PME, « Innovation et organisation des PME », Paris, octobre.

Merle V. (2004), «Un accord historique?», Droit Social, mai 2004.

Michun S. (2000), La petite entreprise et le système. Nature et dynamique des petites entreprises au regard de la ressource humaine, Thèse de Doctorat en Sciences Economiques, université de Montpellier 1.

RERU (1991), «Milieux innovateurs: réseaux d'innovation », Revue d'Économie Régionale et Urbaine, $\mathrm{n}^{\circ} 3 / 4$.

RERU (1993), "Économie de proximité », Revue d'Économie Régionale et Urbaine, $\mathrm{n}^{\circ} 3$.

RERU (1999), « Le paradigme de milieu innovateur dans l'économie spatiale contemporaine", Revue d'Économie Régionale et Urbaine, $\mathrm{n}^{\circ} 3$.

Richardson G.B. (1972), «The Organization of Industry », Economic Journal, Vol. 82, n 327, pp. 883-896.

Salais R., Storper M. (1993), Les mondes de production, Paris, Éditions de l'EHESS.

Thévenot L. (1993), «La trame des organisations », in Globokar T. (Dir.), Entreprise, Société, Communauté, Paris, Autrement, pp. 51-72.

Torrès O. (Dir.) (1998), PME : de nouvelles approches, Paris, Économica.

Torrès O. (2003), « Petitesse des entreprises et grossissement des effets de proximité », Revue Française de Gestion, vol. 29, n 144, mai-juin, pp. 119-138.

Trouvé Ph. (1997), "L'hotellerie-restauration», in Bentabet et al. (1997), Pratiques et représentations de la formation professionnelle dans les très petites entreprises. Approche sectorielle, Marseille, CEREQ, Doc. Multigr., pp. 243-342. 
Résumé

\section{Petites entreprises et territoire, un lien surestimé ?}

Stéphane Michun

Le cas de l'accès des petites entreprises à la formation professionnelle continue (FPC) est emblématique de la survalorisation du territoire. Face à l'incapacité du système de FPC français à réduire significativement les inégalités d'accès à la formation au profit des salariés des petites entreprises, il semble aller de soi qu'il suffit de démultiplier au niveau local les intermédiaires de l'emploi et de la formation, en y déclinant les "bonnes pratiques " observées dans les entreprises les plus formatrices. Nous montrerons que ce type de démarches néglige d'une part, les spécificités et la diversité des petites entreprises, et d'autre part, l'importance des relations affinitaires (irréductibles à la seule proximité spatiale) pour les petites entreprises traditionnelles (de loin les plus nombreuses).

\section{Mots clés}

Accès à la formation, TPE, PME-PMI, approche locale, formation professionnelle en entreprise.

Journal of Economic Literature: M 53 (training), M 51 
